\title{
Performance pondérale et caractéristiques des carcasses des poulets de chair alimentés avec des rations alimentaires à base de graines de Mucuna pruriens
}

\author{
André Boya ABOH*, Serge E. P. MENSAH, Ghislaine S. Théodora ATCHADE \\ et Guy Apollinaire MENSAH
}

\author{
${ }^{1}$ Laboratoire des Recherches Zootechnique, Vétérinaire et Halieutique (LRZVH), Centre de Recherches \\ Agricoles d' Agonkanmey (CRA-Agonmkanmey), Institut National des Recherches Agricoles du Bénin (INRAB), \\ 01 BP2359 Recette Principale, Cotonou, République du Bénin. \\ *Corresponding author,E-mail: aboh.solex@gmail.com; a2abohboya@yahoo.fr
}

\section{RESUME}

L'expansion de la culture de Mucuna pruriens au Bénin a eu comme conséquences la production d'une grande quantité de graines peu valorisées. Ainsi, en alimentation animale, des graines de $M$. pruriens issues des traitements physico-chimiques Rt : graines de $M$. pruriens trempées, décortiquées, bouillies et séchées et Rd : graines de $M$. pruriens décortiquées à sec, trempées, bouillies, retrempées et séchées sont incorporées à trois niveaux 12,$5 ; 18,75$ et $30 \%$ dans des rations alimentaires de poulets. Ces rations sont comparées à la ration témoin (R0) sans la farine de graines de M. pruriens. L'expérimentation a concerné 247 poussins de chair répartis en 13 lots de 19 poussins affectés au hasard aux rations alimentaires. La ration témoin a donné les meilleures performances chez les poulets. Elle est suivie des rations alimentaires $\operatorname{Rt}_{12,5}$ et $\operatorname{Rd}_{12,5}$ en termes de consommation alimentaire $(84,6 \mathrm{~g} / \mathrm{j})$, gain de poids moyen quotidien $(20,8 \mathrm{~g} / \mathrm{j})$ indice de consommation $(4,1$ gMS/gPV). Le poids vif du poulet, de la carcasse et des abats diminuent significativement avec l'accroissement du taux d'inclusion de la farine de graines de $M$. pruriens dans les aliments. Il sera conseillé aux éleveurs de ne pas dépasser la proportion de $12,5 \%$ de la farine de graines de $M$. pruriens pré-traitées $\mathrm{Rd}$ et Rt dans la ration alimentaire des poulets de chair en attendant des investigations avancées.

(C) 2011 International Formulae Group. All rights reserved.

Mots clés : Taux optimal, ration alimentaire, indice de consommation, carcasse, Bénin.

\section{INTRODUCTION}

Le développement des entreprises de volaille a été décrit comme la stratégie la plus rapide pour améliorer le déficit en protéines animales dans les pays du tiers monde, à cause du taux de reproduction élevé et de l'efficacité économique conséquente (Dipeolu et al., 2004). Cependant, au Bénin, le coût de l'alimentation de la volaille ne cesse de s'accroître avec pour corollaire l'augmentation du coût des produits de volaille (viande et œufs). Cette situation indique le besoin et la nécessité d'explorer l'utilisation des ingrédients alimentaires alternatifs qui sont meilleur marché, localement disponible et d'accès aux producteurs. Dans cette perspective, il y a lieu d'évaluer l'adéquation alimentaire de tels produits. Mucuna pruriens est une légumineuse tropicale dont la qualité alimentaire est comparable à celle du soja, parce que contenant des proportions semblables de protéines, de lipide et de 
minéraux. Les graines de $M$. pruriens contiennent environ $32 \%$ de protéines brutes qui renferment presque tous les acides aminés (Ravindran et Ravindran, 1988 ; Dossa et al., 1998) utiles pour les animaux monogastriques. L'adoption et l'expansion de sa culture au Bénin où elle est utilisée dans la lutte contre Imperata cylindrica et comme engrais vert, ont eu comme conséquence la production d'une grande quantité de graines dont seulement une petite proportion est utilisée comme semences. Néanmoins, la forte teneur des graines en facteurs antinutritionnels (FANs) tels que les composés phénoliques, les tannins et les inhibiteurs de protéase peuvent réduire l'utilisation nutritive chez les animaux et les humains (Ravindran et Ravindran, 1988 ; Dossa et al., 1998 ; Carew et al., 2003). Par conséquent, l'adéquation d'un traitement approprié des graines crus est indispensable pour leur valorisation. Un nombre limité d'études ont indiqué que seul le traitement thermique sec (Del Carmen et al., 1999 ; Tuleun et al., 2008) des graines cassées avant le trempage et la cuisson (Dossa et al., 1998 ; Emenalom, 2004 ; Tuleun et Patrick, 2007) ont donné la désintoxication partielle. Toutefois, certaines études mettent en exergue l'interaction du traitement de $M$. pruriens et l'amélioration de la qualité du régime alimentaire dans les systèmes d'élevage de poulet de chair à petite échelle.

L'objectif de notre étude était d'apprécier la réponse à l'incorporation progressive de la farine de graines de $M$. pruriens préalablement traitées à la chaleur, comme ressource alimentaire chez les poulets de chair aussi bien au démarrage qu'à la finition.

\section{MATERIEL ET METHODES Composition de la ration}

Deux modes de traitement physicochimique des graines de $M$. pruriens sont testés (Dossa et al., 1998). Le mode Rt a consisté à tremper les graines de $M$. pruriens dans de l'eau potable pendant 24 heures, égoutter, dépelliculer, bouillir dans l'eau pendant 1 heure, égoutter à nouveau, laisser refroidir et sécher pendant 48 heures à la température ambiante. Pour le mode $\mathrm{Rd}$, les graines de $M$. pruriens sont dépelliculées à sec, trempées dans de l'eau potable pendant 24 heures, égouttées, bouillies dans l'eau pendant une demi-heure, égouttées de nouveau, refroidies à l'air libre, retrempées dans de l'eau potable pendant 24 heures, égouttées et séchées pendant 48 heures à la température ambiante.

Les farines des graines de M. pruriens issues des deux modes de traitement $\mathrm{Rt}$ et $\mathrm{Rd}$ sont incorporées à trois niveaux $12,5 \%$, $18,75 \%$ et $30 \%$ dans les rations expérimentales respectives en remplacement du tourteau de soja (Tableau 1). Ces rations sont comparées à la ration témoin (R0) exempte de farine de graines de $M$. pruriens. Les rations contenant la farine de graines de M. pruriens étaient à deux répétitions et la ration témoin sans répétition. Les rations sont formulées pour la phase de démarrage et de croissance.

\section{Alimentation des poussins}

Un nombre de 247 poussins de chair de Souche ANAK 2000, âgés de cinq jours et d'un poids moyen de $55 \mathrm{~g}$ sont répartis en 13 lots de 19 poussins. Chaque lot est affecté au hasard à une ration à base de farine de graines de $M$. pruriens et à la ration témoin. L'expérimentation a duré 12 semaines décomposées en une phase de démarrage (deuxième à la troisième semaine) et en phase de croissance (quatrième à la douzième semaine). Chaque lot de poussins est élevé sur une litière de copeaux de bois dans des enclos grillagés de $3 \mathrm{~m}^{2}$. Les aliments servis et refusés sont pesés par semaine. Les poussins sont abreuvés ad libitum. Les poulets sont pesés par semaine. Deux poulets par lot sont choisis au hasard et abattus à 12 semaines d'âge afin de faire l'étude des carcasses et des abats. Elle a consisté à la prise de poids, à l'appréciation de la couleur, de la consistance 
A. B. ABOH et al. / Int. J. Biol. Chem. Sci. 5(6): 2306-2316, 2011

Tableau 1 : Composition centésimale des rations alimentaires de démarrage et de croissance des poulets de chair.

\begin{tabular}{|c|c|c|c|c|c|c|c|c|}
\hline \multirow[t]{2}{*}{ Constituants alimentaires } & \multicolumn{5}{|c|}{ Ration de démarrage } & \multicolumn{3}{|c|}{ Ration de croissance } \\
\hline & $\mathbf{R}_{\mathbf{0}}$ & $\begin{array}{c}\mathbf{R t}_{12,5} \\
\text { et } \\
\mathbf{R d}_{12,5} \\
\end{array}$ & $\begin{array}{c}\mathbf{R t}_{18,75} \\
\text { et } \\
\mathbf{R d}_{\mathbf{1 8 , 7 5}} \\
\end{array}$ & $\begin{array}{c}\mathbf{R t}_{30} \\
\text { et } \\
\mathbf{R d}_{30} \\
\end{array}$ & $\mathbf{R}_{\mathbf{0}}$ & $\begin{array}{c}\mathbf{R t}_{12,5} \\
\text { et } \\
\mathbf{R d}_{12,5} \\
\end{array}$ & $\begin{array}{c}\mathbf{R t}_{18,75} \\
\text { et } \\
\mathbf{R d}_{18,75} \\
\end{array}$ & $\begin{array}{c}\mathbf{R t}_{\mathbf{3 0}} \\
\text { et } \\
\mathbf{R d}_{\mathbf{3 0}} \\
\end{array}$ \\
\hline Farine de $M$. pruriens & 00 & 12,5 & 18,75 & 30 & 00 & 12,5 & 18,75 & 30 \\
\hline Farine de soja & 30 & 17,5 & 11,25 & 00 & 30 & 17,5 & 11,25 & 00 \\
\hline Maïs & 40 & 40 & 40 & 40 & 44 & 44 & 44 & 44 \\
\hline Son de blé & 10 & 10 & 10 & 10 & 8 & 8 & 8 & 8 \\
\hline Tourteaux d'arachide & 14 & 14 & 14 & 14 & 12 & 12 & 12 & 12 \\
\hline Tourteaux palmiste & 03 & 03 & 03 & 03 & 03 & 03 & 03 & 03 \\
\hline Prémix & 0,5 & 0,5 & 0,5 & 0,5 & 0,5 & 0,5 & 0,5 & 0,5 \\
\hline Coquille d'huître & 02 & 02 & 02 & 02 & 02 & 02 & 02 & 02 \\
\hline Sel de cuisine & 0,5 & 0,5 & 0,5 & 0,5 & 0,5 & 0,5 & 0,5 & 0,5 \\
\hline \multicolumn{9}{|c|}{ Composition chimique calculée } \\
\hline $\begin{array}{l}\text { Energie métabolisable } \\
(\mathrm{kcal} / \mathrm{kg})\end{array}$ & 2733 & 2388 & 2422 & 1903 & 2780 & 2434 & 2261 & 1949 \\
\hline Protéines Brutes (\% MS) & 27,4 & 21,1 & 21,74 & 12,4 & 26,4 & 20,16 & 17 & 11,41 \\
\hline Fibre totale $(\% \mathrm{MS})$ & 6,1 & 5,94 & 5,93 & 5,79 & 5,69 & 5,59 & 5,55 & 5,47 \\
\hline Calcium (\% MS) & 0,92 & 0,95 & 0,95 & 0,99 & 0,92 & 0,94 & 0,96 & 0,98 \\
\hline Phosphore (\% MS) & 0,54 & 0,5 & 0,51 & 0,45 & 0,51 & 0,47 & 0,46 & 0,42 \\
\hline
\end{tabular}

$\mathrm{R}_{1875}=$ Ration contenant $18,75 \%$ de farine de graines de $M$. pruriens dans la ration alimentaire

$\mathrm{R}_{30}=$ Ration contenant $30 \%$ de farine de graines de $M$. pruriens dans la ration alimentaire

Rt : graines de $M$. pruriens trempées, dépelliculées, bouillies et séchées

Rd : graines de M. pruriens dépelliculées à sec, trempées, bouillies, retrempées et séchées 
et du volume grâce à l'observation visuelle et au toucher de chaque carcasse et abat.

\section{Analyse statistique}

Le logiciel SAS (SAS, 2000) est utilisé pour l'analyse statistique des données. Ainsi, une analyse de variance suivie du test de Bonferroni est réalisée sur les données de mode de traitements des graines de $M$. pruriens et sur celles de leurs niveaux d'incorporation dans les rations alimentaires. Par ailleurs, le test de simultanéité de Dunnett est utilisé pour comparer les rations alimentaires expérimentales à la ration témoin.

\section{RESULTATS}

Effet du mode de traitement des graines de M. pruriens sur la consommation alimentaire, les performances pondérales et les carcasses

Les poulets soumis aux rations du mode de traitement $\mathrm{Rd}$ ont consommé significativement plus d'aliment $(\mathrm{P}<0,01)$ que ceux soumis aux rations du mode traitement Rt. De même, la croissance était significativement plus élevée $(\mathrm{P}<0,01)$ chez les poulets soumis aux rations du mode de traitement $\mathrm{Rd}$ que ceux soumis aux rations du mode traitement Rt (Tableau 2). Par contre, l'indice de consommation alimentaire était similaire pour les rations alimentaires issues des modes de traitements de graines de $M$. pruriens $\mathrm{Rt}$ et $\mathrm{Rd}$ chez les poulets. Les rations alimentaires issues des modes de traitements des graines de $M$. pruriens $\mathrm{Rt}$ et $\mathrm{Rd}$ ont eu des effets similaires $(\mathrm{P}>0,05)$ sur les caractéristiques des carcasses et des abats de poulets de chair (Tableau 3).

Effet du taux d'incorporation des graines de $M$. pruriens dans les rations alimentaires sur l'ingestion alimentaire, les performances pondérales et les carcasses

En fonction du taux d'incorporation des graines de $M$. pruriens, dans les rations, la consommation des rations alimentaires a varié de 60 à $84,5 \mathrm{~g} / \mathrm{j}$, le gain de poids a varié de 10,8 à $20,8 \mathrm{~g} / \mathrm{j}$ et l'indice de consommation a varié de 4,1 à 5,6 chez les poulets (Tableau 4). Les résultats ont indiqué une différence significative $(\mathrm{p}<0,01)$ entre les rations alimentaires expérimentales contenant différents taux d'incorporation de $M$. pruriens. La consommation, le gain de poids moyen quotidien et l'indice de consommation étaient meilleurs $(\mathrm{P}<0,05)$ chez tous les poulets alimentés avec la ration contenant $12,5 \%$ de farine de graines de $M$. pruriens traitées Rt et Rd (Tableau 4). Concernant l'étude des caractéristiques de carcasse, les poids vifs moyens, les poids des carcasses et des abats des poulets de chair sont significativement plus élevés $(\mathrm{P}<0,001)$ pour les rations alimentaires contenant $12,5 \%$ de farine de graines de M. pruriens (Tableau 5).

Effets des rations alimentaires contenant ou non différents niveaux de la farine de graines de $M$. pruriens sur la consommation et les performances pondérales

Les résultats montrent que la ration témoin a donné la meilleure performance chez les poulets $(\mathrm{P}<0,05)$ en termes de consommation alimentaire $(110,1 \mathrm{~g} / \mathrm{j})$, gain de poids quotidien $(34,3 \mathrm{~g} / \mathrm{j})$, indice de consommation (3,3 gMS/gPV) (Tableau 6). Cette performance est suivie respectivement de celles des poulets alimentés aux rations $\mathrm{Rt}_{12,5}$ et $\mathrm{Rd}_{12,5}$. Les autres rations ont données des performances faibles.

Les résultats ont indiqué une meilleure performance $(\mathrm{p}<0,05)$, des caractéristiques des carcasses et des abats de poulets alimentés avec la ration témoin dépourvue de graines de M. pruriens (Tableau 7). Au sein des rations alimentaires contenant la farine de graines $M$. pruriens, le poids vif, le poids de la carcasse et des abats ont diminué significativement $(\mathrm{P}<0,05)$ avec l'accroissement du taux de graines de $M$. pruriens dans les aliments quelque soit le mode de traitement des graines de $M$. pruriens. 
A. B. ABOH et al. / Int. J. Biol. Chem. Sci. 5(6): 2306-2316, 2011

Tableau 2 : Consommation alimentaire moyenne, gain moyen quotidien et indice de consommation (g MS/g poids vif) en fonction des modes de traitement des graines de M. pruriens chez les poulets de chair.

\begin{tabular}{lccc}
\hline Paramètres & Modes de traitement des graines & Erreur-Type de la moyenne \\
\hline & Rt & Rd & \\
Consommation alimentaire (g MS/j) & 65,04 & 81,46 & $2,34 * * *$ \\
Gain moyen quotidien (g) & 14,17 & 17,61 & $0,55^{* * *}$ \\
Indice de consommation & $4,60: 1$ & $4,63: 1$ & $\mathrm{~ns}$ \\
\hline$* * * * \mathrm{p}$
\end{tabular}

*** $=\mathrm{p}<0,001 ; \mathrm{ns}=$ non significative

Rt : graines de $M$. pruriens trempées, dépelliculées, bouillies et séchées

Rd : graines de M. pruriens dépelliculées à sec, trempées, bouillies, retrempées et séchées.

Tableau 3 : Effet des modes de traitement des graines de $M$. pruriens incorporées dans les rations alimentaires sur le poids vif corporel, le poids de la carcasse, les abats et le sang des poulets de chair.

\begin{tabular}{lccc}
\hline Variables & \multicolumn{2}{c}{ Modes de traitement } & Erreur-type de la moyenne \\
\hline & $\mathrm{Rt}$ & $\mathrm{Rd}$ & \\
Poids vif $(\mathrm{g})$ & 950 & 983 & 58 \\
Carcasse $(\mathrm{g})$ & 567 & 567 & 47,3 \\
Rendement carcasse $(\%)$ & 56,9 & 57,8 & 1,6 \\
Sang (ml) & 31 & 34 & 1,8 \\
Plume (g) & 83 & 69 & 6,6 \\
Patte + têtes (g) & 123 & 104 & 6,1 \\
Viscères (g) & 175 & 194 & 10,9 \\
Viande (g) & 363 & 377 & 30,2 \\
Os (g) & 204 & 200 & 19 \\
\hline
\end{tabular}

Rt : graines de M. pruriens trempées, dépelliculées, bouillies et séchées

$\mathrm{Rd}$ : graines de $M$. pruriens dépelliculées à sec, trempées, bouillies, retrempées et séchées, $\mathrm{P}>0,05$. 
A. B. ABOH et al. / Int. J. Biol. Chem. Sci. 5(6): 2306-2316, 2011

Tableau 4 : Effet du taux d'incorporation des graines de $M$. pruriens dans la ration sur la consommation alimentaire, le gain moyen quotidien et l'indice de consommation (g MS/g poids vif) chez des poulets de chair.

\begin{tabular}{lcccc}
\hline Paramètres & $\begin{array}{c}\text { Proportion (\%) de } \boldsymbol{M} \text {. pruriens } \\
\text { dans les rations }\end{array}$ & $\begin{array}{c}\text { Erreur-Type de la } \\
\text { différence }\end{array}$ \\
\hline & 12,5 & 18,75 & 30 & \\
Consommation alimentaire (g MS/j) & 84,56 & 76,25 & 59,94 & $2,34 * * *$ \\
Gain moyen quotidien (g) & 20,84 & 16,08 & 10,76 & $0,953 * * *$ \\
Indice de consommation & $4,06: 1$ & $4,74: 1$ & $5,57: 1$ & $0,241 * * *$ \\
\hline$* * *=p<0,001$ & &
\end{tabular}

$* * *=\mathrm{p}<0,001$

Tableau 5 : Effet du taux d'incorporation des graines de M. pruriens dans les rations alimentaires sur le poids vif, la carcasse et les abats des poulets de chair.

\begin{tabular}{lcrrrr}
\hline Variables & \multicolumn{2}{c}{$\begin{array}{c}\text { Proportion } \\
\text { pruriens dans les rations }\end{array}$} & $\begin{array}{c}\text { de } \\
\text { M. }\end{array}$ & $\begin{array}{c}\text { Erreur-Type de la } \\
\text { moyenne }\end{array}$ \\
\hline & 12,5 & 18,75 & 30 & & \\
Poids vif (g) & 1200 & 950 & 750 & $71,0^{*}$ \\
Carcasse (g) & 738 & 569 & 394 & $57,9^{*}$ \\
Rendement carcasse (\%) & 60,3 & 59,4 & 52,4 & $2,0^{*}$ \\
Sang (ml) & 40 & 33 & 26 & $2,2^{*}$ \\
Plume (g) & 88 & 78 & 63 & $8,1^{*}$ \\
Pattes+ têtes (g) & 125 & 113 & 103 & $7,4^{*}$ \\
Viscères (g) & 222 & 169 & 163 & $13,4^{*}$ \\
Viande (g) & 494 & 378 & 238 & $37,0^{*}$ \\
Os (g) & 263 & 188 & 156 & $23,2^{*}$ \\
\hline
\end{tabular}


Tableau 6 : Consommation alimentaire, gain de poids moyen quotidien et indice de consommation en fonction des niveaux d'incorporation et des modes de traitement.

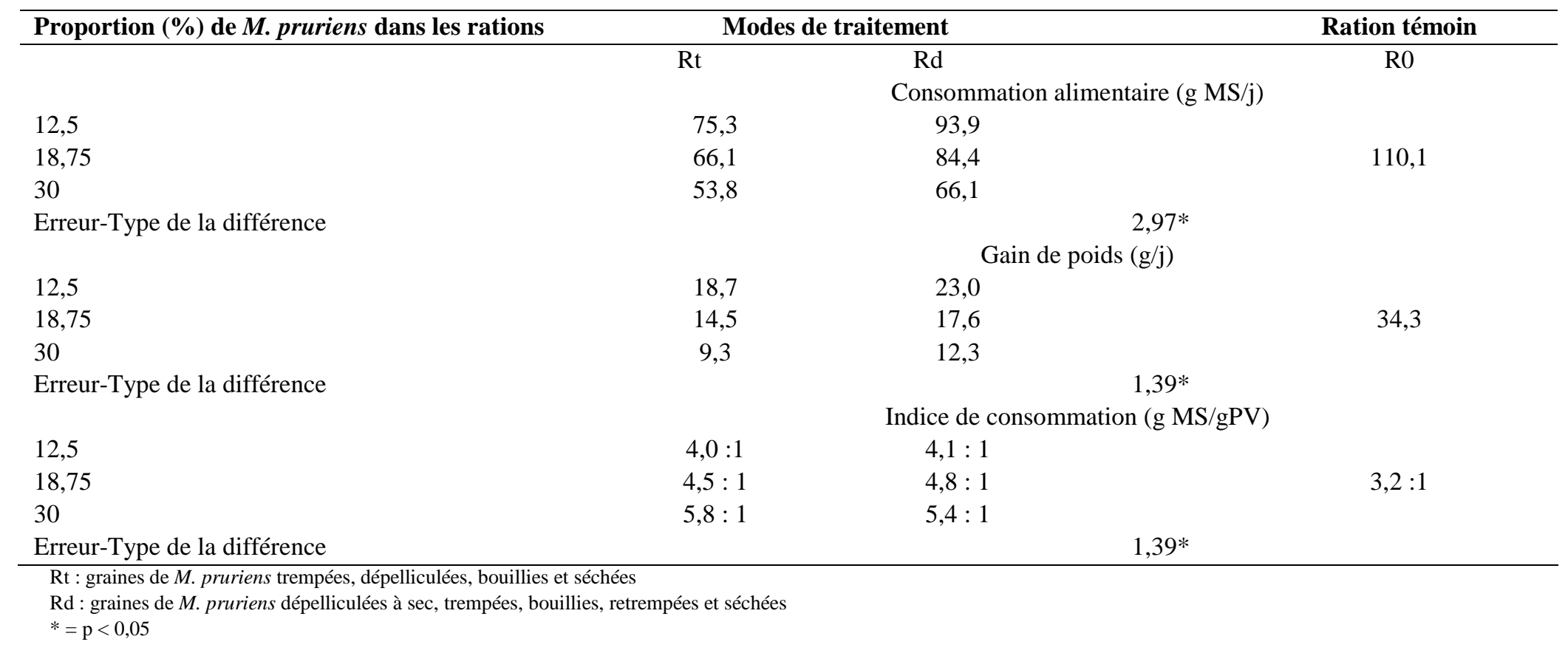


Tableau 7 : Caractéristiques de carcasse et abats des poulets en fonction des niveaux d'incorporation et des modes de traitement de graines de M. pruriens dans les rations alimentaires.

\begin{tabular}{|c|c|c|c|c|c|c|c|c|}
\hline \multirow[t]{2}{*}{ Variables } & \multicolumn{7}{|c|}{ Rations alimentaires } & \multirow{2}{*}{$\begin{array}{c}\text { Erreur-Type de } \\
\text { la différence } \\
\end{array}$} \\
\hline & $\overline{\mathbf{R}_{\mathbf{0}}}$ & $\mathbf{R t}_{12,50}$ & $\mathbf{R d}_{12,50}$ & $\mathbf{R t}_{18,75}$ & $\operatorname{Rd}_{18,75}$ & $\mathbf{R t}_{\mathbf{3 0}}$ & $\mathbf{R d}_{\mathbf{3 0}}$ & \\
\hline Poids vif (g) & 1950 & 1175 & 1225 & 925 & 975 & 850 & 650 & $172,6^{*}$ \\
\hline Carcasse (g) & 1275 & 688 & 788 & 550 & 588 & 463 & 325 & $140,7^{*}$ \\
\hline Rendement (\%) & 65,5 & 57,6 & 63,1 & 58,8 & 59,9 & 54,3 & 50,5 & $2,80 *$ \\
\hline Sang (ml) & 64,5 & 35,8 & 43,3 & 30,5 & 34,5 & 26,8 & 25,5 & $5,26^{*}$ \\
\hline Plume (g) & 125 & 100 & 75 & 87,5 & 68,8 & 62,5 & 62,5 & 20,93 \\
\hline Pattes + têtes (g) & 200 & 131,3 & 118,8 & 125 & 100 & 112,5 & 93,8 & $18,08^{*}$ \\
\hline Viscères $(\mathrm{g})$ & 300 & 200 & 243,8 & 162,5 & 175 & 162,5 & 162,5 & $21,79 *$ \\
\hline Viande $(\mathrm{g})$ & 950 & 462 & 525 & 350 & 406,3 & 275 & 200 & $89,37 *$ \\
\hline Os $(\mathrm{g})$ & 325 & 225 & 300 & 200 & 175 & 187 & 125 & $50,90 *$ \\
\hline $\begin{array}{l}\text { Rt : graines de } M . \\
\text { Rd : graines de } M . \\
12,5=12,5 \% \text { de } \\
18,75=18,75 \% \text { de } \\
30=30 \% \text { de farine } \\
*=p<0,05 \\
\text { Viscère = intestin. }\end{array}$ & $\begin{array}{l}\text { } 2 s \text { trempées, d } \\
n s \text { dépelliculé } \\
\text { e graines de } I \\
\text { de graines de } \\
\text { aines de } M . p r\end{array}$ & $\begin{array}{l}\text { culées, bouill } \\
\text { ec, trempées, } \\
\text { triens dans la } \\
\text { ruriens dans } 1 \\
\text { s dans la ratio } \\
\text { eur. }\end{array}$ & $\begin{array}{l}\text { séchées } \\
\text { llies, retrempé } \\
\text { n alimentaire } \\
\text { ion alimentair } \\
\text { mentaire }\end{array}$ & séchées & & & & \\
\hline
\end{tabular}




\section{DISCUSSION}

Les modes de traitement $\mathrm{Rt}$ et $\mathrm{Rd}$ ne sont pas aussi efficaces pour induire un aliment capable d'améliorer la performance pondérale similaire à celle induite par la ration témoin sans farine de graine de $M$. pruriens. Certains facteurs anti-nutritionnels (FANs) comme la L-dopa sont solubles dans l'eau et s'oxydent facilement au contact de l'air (Budavari, 1989) ; d'autres comme l'inhibiteur de la trypsine, l'inhibiteur de la protéase, le tannin, l'oxalate et l'acide cyanhydrique sont éliminés par ébullition (Van der Poel, 1989 ; Tuleun et al., 2009). Les études précédentes ont démontré la nécessité de prétraiter la graine de $M$. pruriens à la chaleur associée ou non au trempage dans l'eau puis séchage avant son utilisation comme ressource alimentaire des monogastriques (Dossa et al., 1998; Iyayi and Taiwo, 2003; Farougou et al., 2006, Dahouda et al., 2009b). Il ressort des deux modes de traitement des graines $M$. pruriens, l'élimination probable des mêmes types de FANs à travers la combinaison des opérations de trempage, d'ébullition et de séchage à l'air ambiant. Ce qui expliquerait les performances pondérales similaires observées chez les poulets alimentés aux rations $\mathrm{Rt}$ et $\mathrm{Rd}$.

Les résultats de cette recherche révèlent que les poulets semblent tolérer $12,5 \%$ de taux d'incorporation de la farine de graines de $M$. pruriens issue des traitements $\mathrm{Rt}$ et $\mathrm{Rd}$ dans l'aliment. Ce taux d'incorporation induit un indice de consommation proche $(\mathrm{p}<0,05)$, mais pas suffisant pour atteindre celui de la ration témoin. Ainsi, en alimentation des poulets de chair, le taux d'incorporation optimale des graines de $M$. pruriens traitées par les deux modes testés ici est de $12,5 \%$. En effet, l'augmentation des taux d'incorporation de la farine de graines de $M$. pruriens traitées induit une diminution de la consommation alimentaire, de la croissance pondérale, du rendement de la carcasse et des abats, et une augmentation de l'indice de consommation. La faible croissance et la baisse des poids des carcasses et des abats enregistrées chez les poulets dont les rations alimentaires contenaient plus de $12,5 \%$ de graines de $M$. pruriens traitées seraient liées aux effets toxiques des FANs résiduels après le traitement des graines. Ces FANs présents dans la graine de $M$. pruriens perturbent le tractus digestif ainsi que le fonctionnement des organes (Iyayi et Taiwo, 2003). Cependant, avec la méthode de traitement des graines utilisée, nos résultats ne concordent pas avec l'incorporation de taux de $20 \%$ de graines de $M$. pruriens trempées et bouillies pendant 40 et $60 \mathrm{mn}$ dans la ration alimentaire de finition suggérée pour obtenir un effet positif sur la croissance pondérale de poulet (Tuleun et al., 2009). Les indices de consommation (IC) moyens obtenus pour les taux de $12,5 \%$ d'incorporation des graines de M. pruriens traitées sont inférieurs à celui précédemment enregistré avec le même taux pour des graines traitées à l'eau chaude (IC = $4,94 \mathrm{~g} \mathrm{MS} / \mathrm{gPV}$ ) au cours de la phase de démarrage (Iyayi et al., 2006). Toutefois, l'indice de consommation est trop élevé par rapport à celui enregistré lors d'études précédentes $(\mathrm{IC}=2,45$ ) au cours de la phase de finition (Iyayi et al., 2006). L'expérimentation réalisée chez les pintadeaux a révélé que l'aliment à base des graines de $M$. pruriens ayant subit les mêmes traitements ont induit les meilleures performances de croissance chez les pintades comparées à l'aliment témoin (Farougou et al., 2006). Les auteurs ont recommandé aux éleveurs d'utiliser dans la ration alimentaire, la farine de $M$. pruriens traitée $\mathrm{Rt}$ et incorporée à 10,62\% ou traitée Rd incorporée à $13,75 \%$. Par ailleurs, pour une meilleure consommation et une bonne digestibilité chez les pintades, Dahouda et al. (2009a) rapportent un taux d'incorporation de $12 \%$ de graines de $M$. pruriens ayant subies un traitement similaire à celui réalisé pour cette expérimentation. Ces différents taux d'incorporation de $M$. pruriens cuite à l'eau chaude dans l'aliment suggérés par Farougou et al. (2006) et Dahouda et al. (2009a) sont proches de celui enregistré $(12,5 \%)$ qui a donné une meilleure performance pour la présente expérimentation. Toutefois, ces deux 
taux d'incorporation de $M$. pruriens $(10,62$ à $13,75 \%$ ) sont nettement inférieur au taux $20 \%$ suggéré par Dahouda et al. (2009b) pour obtenir un gain de poids élevé chez les pintadeaux. Cette différence de taux d'incorporation des graines traitées suggérée par Dahouda et al. (2009b) est probablement liée aux processus de traitement testés, notamment le matériel et la durée de grillage des graines au four de 100 à $120{ }^{\circ} \mathrm{C}$ pendant $20 \mathrm{mn}$ et la cuisson des graines à l'eau chaude pendant $30 \mathrm{mn}$. Les analyses chimiques effectuées indiquent que le traitement par chauffage modifie la concentration de plusieurs nutriments de graines de $M$. pruriens (Dahouda et al., 2009b). Il est aussi probable que la combinaison du trempage et $\mathrm{du}$ retrempage des graines de $M$. pruriens bouillies modifie la concentration de plusieurs nutriments de la graine de $M$. pruriens. Ainsi, le taux d'incorporation des graines de $M$. pruriens dans l'aliment de la volaille semble être lié à la méthode de traitement. Cependant, par rapport au défi lié à l'utilisation du $M$. pruriens comme ressource alimentaire de volaille, les analyses de la L-Dopa dans des échantillons de foies, de reins et de muscles n'ont indiqué aucune présence des résidus dans le tissu lorsque les graines sont traitées (Daouda et al., 2009b). Par conséquent, selon ces auteurs, les risques de la consommation de la L-Dopa en mangeant de la viande de pintade et probablement de poulets nourris avec du $M$. pruriens traité peut être considéré comme nul.

\section{Conclusion}

Somme toute, au stade actuel de nos recherches, nous pouvons retenir que les poulets de chair sont sensibles à l'efficacité du traitement des graines de $M$. pruriens et à leur taux d'incorporation dans leurs rations alimentaires. Les modes de traitement des graines de $M$. pruriens $\mathrm{Rt}$ et $\mathrm{Rd}$ peuvent être utilisés pour réduire de façon efficace les effets des FANs sur la croissance des poulets de chair. Ainsi, il peut être conseillé aux éleveurs d'utiliser jusqu'à $12,5 \%$ de la farine de graines de $M$. pruriens pré-traitées par le mode Rt ou Rd dans l'aliment en cas de rareté des sources de protéines. Cependant, des investigations plus poussées sur les résidus des facteurs inhibiteurs de croissance des poulets de chair dans les graines traitées de $M$. pruriens s'avèrent indispensable.

\section{REMERCIEMENTS}

Les auteurs remercient sincèrement le Centre Béninois de Recherches Scientifiques et Techniques (CBRST) pour avoir financé ce protocole de recherche.

\section{REFERENCES}

Budavari S. 1989. An Encyclopedia of Chemicals, Drugs and Biologicals. (11th edition). Merck \& Co Inc: Rahway; 861862.

Carew LB, Hardy D, Weis J, Alster F, Mischler SA, Gernat A, Zarkrzewska EI. 2003. Heating raw Velvet beans (Mucuna pruriens) reverses some antinutritional effects on organ growth, blood chemistry and organ histology in growing chickens. Tropical and Sub Tropical Agroecosystems, 1: 267-275.

Dahouda M, Toléba SS, Youssao AKI, Hambuckers A, Dangou-Sapoho R, Martin GB, Fillet M, Hornick J-L. 2009a. Nutrient digestibility of Mucuna (Mucuna pruriens var. utilis) bean in guinea fowl (Numida meleagris, L): Effects of heat treatment and levels of incorporation in diets. British Poultry Science, 50(5): 564572.

Dahouda M, Toleba SS, Youssao AKI, Mama Ali AA, Dangou-Sapoho RK, Ahounou SG, Hambuckers A, Hornick J-L. 2009b. The Effects of Raw and Processed Mucuna pruriens Seed Based Diets on the Growth Parameters and Meat Characteristics of Benin Local Guinea Fowl (Numida meleagris L). International Journal of Poultry Science, 8(9): 882-889.

Dipeolu MA, Adebayo AJ, Oke OM. 2004. Residues of Streptomycin antibiotic in commercial layers in Abeokuta and 
Ibadan metropolis. Nig. J. Anim. Prod., 31: $130-134$.

Dossa CS, Mensah GA, Dossa AD, Adoun C. 1998. Influence de divers traitements physico-chimiques de graines de Mucuna pruriens sur leur composition chimique en nutriments. Tropicultura, 16-17, 141146.

Emenalom OO. 2004. Comparative Performance of broiler chicks fed diets containing differently processed Mucuna Pruriens seed meals. Nig. J. Anim. Prod., 31: 12-16.

Farougou SF, Kpodekon M, Tokannou R, Djossou VD, Akoutey A, Youssao IAK. 2006. Utilisation de la farine de Mucuna pruriens (L.) DC dans l'aliment de croissance des pintades (Numida meleagris). Revue Méd. Vét., 157(10): 502-508.

Iyayi EA, Taiwo VO. 2003. The effects of diets incorporating mucuna (M. pruriens) seed meal on the performance of laying hens and broilers. Tropical and Subtropical Agroecosystems, 1: 239-246.

Iyayi EA, Ososanya TO, Taiwo VO, Adeniji OA. 2006. Growth, haematology and organ histopathology in broilers fed raw and processed velvet bean-based diets. Conference on International Agricultural Research for Development Tropentag 2006 University of Bonn, October 11-13, 2006.
Ravindran V, Ravindran G. 1988. Nutritional and anti-nutritional characteristics of Mucuna (Mucuna utilis) bean seeds. J. Sci. Agri., 46: 71-79.

Tuleun CD, Patrick JP. 2007. Effect of duration of cooking Mucuna utilis seed on proximate composition, levels of anti nutritional factors and performance of broiler chickens. Nig. J. Anim. Prod., 34: 45-53.

Tuleun CD, Carew SN, Ajiji I. 2008. Feeding value of velvet beans (Mucuna utilis) for laying hens. Livest. Res. Rural Dev., 20(5).

Tuleun CD, Patrick JP, Tiamiyu LO. 2009. Evaluation of Raw and Boiled Velvet Bean (Mucuna utilis) as Feed Ingredient for Broiler Chickens. Pakistan Journal of Nutrition, 8(5): 601-606.

SAS INSTITUTE. 2000. SAS/ STAT User's Guide. Version 9.5, SAS Institute, Cary, North Carolina, USA.

Van Der Poel AFB. 1989. Effects of processing on antinutritional factors (ANF) and nutritional value of legume seeds for non-ruminant feeding. In Recent Advances of Research in Antinutritional Factors in Legume Seeds, Huisman J, van der Poel AFB (eds). Pudoc: Wageningen; 213-229. 